\title{
A Review Paper on Image Forgery Detection In Image Processing
}

\author{
Shivani Thakur ${ }^{1}$ Ramanpreet Kaur ${ }^{2}$ Dr. Raman Chadha ${ }^{3}$ Jasmeet $_{\text {Kaur }}{ }^{4}$ \\ ${ }^{1,4}$ M.tech student, CGCTC, Jhanjeri, Mohali \\ ${ }^{2}$ Assistant Professor CGCTC, Jhanjeri, Mohali \\ ${ }^{3}$ Professor, Head(CSE), Jhanjeri, Mohali
}

\begin{abstract}
The image forensics is the technique which is applied to hide image important information. In the base paper, the technique of SIFT algorithm is applied to mark the objects in the image. In the SIFT algorithm whole image is scanned and from the scanned image objects are marked. The properties of the marked object are accessed and objects which have similar properties are classified into group and other are into second. To classify the similar type of objects techniques like block based \& Key Point based technique, shift key point can be used.
\end{abstract}

Keywords: Image Processing, image forensics, digital signature, image preprocessing.

\section{Introduction}

A digital image is defined as a 2D function i.e. $\mathrm{f}(\mathrm{x}, \mathrm{y})$ where $(\mathrm{x}, \mathrm{y})$ shows the spatial coordinates and the amplitude of $f$ at any point $(\mathrm{x}, \mathrm{y})$ known as the intensity or gray level of image at that particular point. In case when $\mathrm{x}, \mathrm{y}$ and the amplitude value of $\mathrm{f}$ are finite and discrete quantities, we can call that image as a digital image. Basically, an image i.e. processed by a digital computer is called as a digital image. It may be noted that a digital image consists of a finite number of elements which are having a particular location and value. These elements of image are known as image elements, picture elements or pixels.

Image processing is a technique in which the image is converted into digital form and some operations are performed on it for the purpose of getting and enhanced image or extracting some meaningful or useful information from it. It is a kind of signal processing in which the input may be image e.g. video frame, photograph and output may possibly be the image or some characteristics related with the image. Image processing involves the following three steps:

1. Involves importing the image via using image acquisition tool which may be optical scanner or digital photography.

2. Now the image is analyzed and manipulated which involves data compression and image enhancement.

3. Last stage is output which involves the result i.e. altered image. There are two methods that are used for image processing:

i. Analog image processing

ii. Digital image processing

i. Analog image processing

It may be used for hard copies i.e. printouts or photographs etc.

\section{ii. Digital image processing}

It is the process of manipulating the digital image using computers.

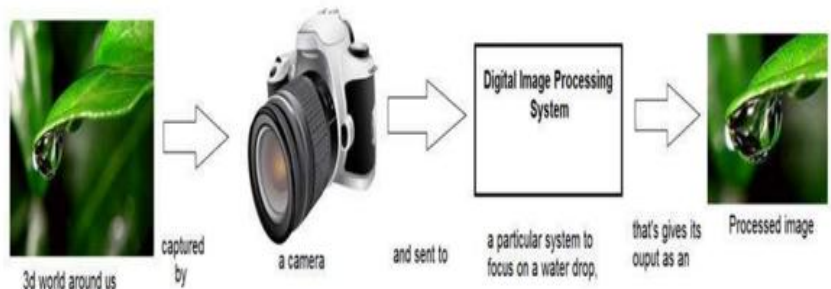

Fig1. Digital image processing

\section{Literatute Review}

This review paper provides a survey on different image forensics techniques which are given in previous papers. In this paper [1], they have proposed methodologies to identify these images and find out the fake region of the forgery image. They have developed algorithm depend upon the concept of abnormal anomalies and identify the forgery regions. 
In this paper [2], first of all classification of image forgery detection technique has been discussed. After that pixel based detection is also defined. A technique for copy-move forgery detections discussed. But this technique is applied on shifted region only. Proposed technique is developed to cover all the limitation of the both the techniques and fast copy moving technique.

In this paper [3], they reviewed the technique to remove shift key point attacks from the image for the betterment of SIFT-based copy-move forgery detection. To handle these types of attacks three forensic techniques have discussed in this paper. They apply the detectors to a practical image forensic scenario of SIFT based copy-move forgery detection to assume the SIFT-based detectors and as a result give new proposed technique.

In this paper [4], they explained that it is very easy to tamper the images. Image forensic can be used to determine the protection of the image. They have discussed the various copy move forgery detection techniques which includes Block based \& Key Point based techniques.

In this paper [7], they discussed passive approach to detect digital forgeries by checking its artifact consistencies. JPEG image compression could be used as a natural authentication code for blocking artifact. A technique has been proposed based on estimated quantized table using power spectrum of the DCT coefficient histogram. Experiments have been done to validate proposed techniques and in future discovery of other image quality consistencies is measured.

In this paper [6] they classified the image forgery detection technique. Later on they also discussed two important techniques for the pixel based forgery detection. In this paper they also have discussed copy-move forgery detection technique. This technique only deals with shifting of copied regions. So there is a need of another technique for fast copy move detection and it is also discussed in this paper. At the end both techniques are compared and analyzed.

\section{Various Techniques Of Image Processing}

Various techniques of the image processing are:

1. Image Representation

2. Image Preprocessing

3. Image Enhancement

4. Image Analysis

5. Image Segmentation

6. Image Restoration

7. Image Compression

\section{Image Representation}

In the real world image is defined as a function of two real variables. We can say that $f(x, y)$ with $f$ as the amplitude or brightness of the image at the real coordination position $(\mathrm{x}, \mathrm{y})$. In most cases $\mathrm{f}(\mathrm{x}, \mathrm{y})$ which is lying on the face of the sensors. Typically an image file such as JPEG, BMP, TIFF etc [2], has some header and picture information. A header generally consists details like format identifier, number of bits/pixel, resolution, compression type etc.

\section{Image Preprocessing}

The main aim of preprocessing is an improvement of image data which is used to remove unwanted images and distortions from the images for image processing.

\section{Image Enhancement}

Sometimes images which are obtained from satellites and cameras have poor quality of brightness and contrast because limitations of imaging sub system and illumination while capturing image. Image has different types of noise. In image enhancement, the goal is to accentuate certain image features for subsequent analysis or for image display [1,2].

\section{Image Analysis}

Image Analysis is concerned with quantitative measurement of the image to make it perfect and noise free. It requires extraction of certain features for the identification of the object. Segmentation techniques are used to isolate desired objects from the scene to make them perfect [5].

\section{Image Segmentation}

Image segmentation is the process on which image can be divided into number of its subparts. The problem which is being solved is responsible for the division of the object identification. 


\section{Image Restoration}

It is concerned with filtering the observed image to minimize the effect of degradations. Effectiveness of image restoration depends on the extent and accuracy of the knowledge of degradation process as well as on filter design. Image restoration differs from image enhancement in that the latter is concerned with more extraction or accentuation of image features.

\section{Image Compression}

It is concerned with minimizing the no of bits required to represent an image. Application of compression are in broadcast TV, remote sensing via satellite, military communication via aircraft, radar, teleconferencing, facsimile transmission, for educational \& business documents, medical images that arise in computer tomography, magnetic resonance imaging and digital radiology, motion , pictures ,satellite images, weather maps, geological surveys and so on [2].

\section{Challenges In Digital Image Processing}

1. Compression: The image storage and transmission involves the use of digital techniques 2 . Enhancement: In case of enhancement the quality of image is improved. The quality of image may be poor in terms of contrast which may be low, image may be noisy or it may be blurred etc. In order to overcome this, many algorithms have been used .But difficult part is how we can remove the degradation without hurting the signal. A major challenge is in digital image processing is how can we enhance severely degraded images. 3 . Recognition: An image recognition system means to classify input pattern into one of a set of pre-specified classes. This task is easy in case of number of classes is small and if the members in the same class are almost same. But difficulty level increases if the number of classes is large or if the member in same class can look very different.4. Visualization: The main task of visualization is to generate images based on three dimensional object and scene object. The main challenge is how we can model dynamic scenes containing non-grid objects. The model should be realistic and the computation cost should be reasonable.

\section{Image Forgery Detection}

Nowadays it is easy to remove and add some elements from the image for the purpose to manipulate and to get some good results of image forgeries. In image processing, different types of software are used. Some software can change a particular block of image without affecting the originality of the image. This kind of modification cannot be noticed by human eye. The main task is to verify the original image. For the purpose of manipulating an image various techniques of image processing like scaling, rotation, blurring, filtering and cropping can be used. The image forgery detection is required in various fields of image processing. Image forgery detection is raising research field with important applications for ensuring the credibility of digital images.Image processing can be divided into two categories:

a. Active Approach

b. Passive Approach

a. Active Approach: In active approach, some preprocessing of digital image like embedded watermark or signature generation is required at the time of creating image and limits its applications. This approach is not used for authentication purpose.

b. Passive Approach: In the passive approach, digital signature is not used for the purpose of authentication. In this technique some assumptions are used that digital forensics may not leave any visual clue for tempering the image. It may alter the underlying statistics of image. The image forensics tools can be grouped into five categories:

i. Pixel based technique

ii. Format based technique

iii. Camera based technique

iv. Physically based technique

v. Geometric based technique

VI. Comparison Table

\begin{tabular}{|l|l|l|l|}
\hline Author & Year & Description & Outcome \\
\hline S. Murali et al [1] & 2012 & $\begin{array}{l}\text { methodologies to identify these } \\
\text { images and find out the fake } \\
\text { region of the forgery image. }\end{array}$ & $\begin{array}{l}\text { algorithm depend upon the concept of } \\
\text { abnormal anomalies and identify the } \\
\text { forgery regions. }\end{array}$ \\
\hline Andrea Costanzo et.al [3] & 2014 & $\begin{array}{l}\text { To handle shift key point of } \\
\text { attacks three forensic techniques } \\
\text { have discussed. }\end{array}$ & $\begin{array}{l}\text { apply the detectors to a practical image } \\
\text { forensic scenario of SIFT-based copy- } \\
\text { move forgery detection to assume the } \\
\text { SIFT-based detectors }\end{array}$ \\
\hline
\end{tabular}


A Review Paper on Image Forgery Detection In Image Processing

\begin{tabular}{|l|c|l|l|}
\hline $\begin{array}{l}\text { Ms. P. G.Gomase, Ms. N. R. } \\
\text { Wankhade [2] }\end{array}$ & 2014 & pixel based detection is defined & $\begin{array}{l}\text { No method achieved 100\% robustness } \\
\text { against post processing operations. }\end{array}$ \\
\hline Rohini.A.Maind et.al [5] & 2014 & $\begin{array}{l}\text { four features are extracted to } \\
\text { reduce the dimensions }\end{array}$ & $\begin{array}{l}\text { do blurring and remove noise from the } \\
\text { image with low complexity }\end{array}$ \\
\hline $\begin{array}{l}\text { Gagandeep Kaur, Manoj } \\
\text { Kumar [4] }\end{array}$ & 2015 & $\begin{array}{l}\text { various copy move forgery } \\
\text { detection techniques }\end{array}$ & $\begin{array}{l}\text { Block based \& Key Point based } \\
\text { techniques. }\end{array}$ \\
\hline
\end{tabular}

\section{Conclusion}

In order to detect image forgery, an accurate and successful method should be used and this can be done with the help of image processing a approach. This paper reviewed various techniques which have been already used. These techniques are related to segmentation and classification. Using these methods, identification image forgeries has been accurately done but still having some chances for the improvements in the existing techniques.

\section{References}

[1]. S And Basavaraj S. Anami, “Comparison And Analysis Of Photo Image Forgery Detection Techniques", International Journal On Computational Sciences \& Applications (Ijcsa) Vo2, No.6, December 2012

[2]. Ms. P. G.Gomase, Ms. N. R. Wankhade, “Advanced Digital Image Forgery Detection- A Review”, Iosr Journal Of Computer Science (Iosr-Jce) E-Issn: 2278-0661, P-Issn: 2278-8727 Pp 80-83 2014

[3]. Andrea Costanzo, Irene Amerini, Roberto Caldelli, Mauro Barni, "Forensic Analysis Of Sift Keypoint Removal And Injection", Ieee Transactions On Information Forensics And Security, Vol. 9, No. 9, September 2014

[4]. Gagandeep Kaur, Manoj Kumar, "Study Of Various Copy Move Forgery Attack Detection In Digital Images", International Journal Of Research In Computer Applications And Robotics, Vol.3 Issue 9, Pg.: 30-34 September 2015

[5]. Rohini.A.Maind, Alka Khade, D.K.Chitre, "Image Copy Move Forgery Detection Using Block Representing Method", International Journal Of Soft Computing And Engineering (Ijsce) Issn: 2231-2307, Volume-4, Issue-2, May 2014

[6]. Pradyumna Deshpande , Prashasti Kanikar, "Pixel Based Digital Image Forgery Detection Techniques", International Journal Of Engineering Research And Applications (Ijera) Issn: 2248-9622 Vol. 2, Issue 3, May-Jun 2012, Pp. 539-543

[7]. Shuiming Ye, Qibin Sun ,And Ee-Chien Chang, "Detecting Digital Image Forgeries By Measuring Inconsistencies Of Block Artifact", Ieee, 2007

[8]. Preeti Yadav, Yogeshrathore, Aarti Yadav,” Dwt Based Copymove Image Forgery Detection”, International Journal Of Advanced Research In Computer Science An Electronics Engineering Volume 1, Issue 5, July 2012

[9]. Maind. R,Khade.A, Chitre.D, "Image Copy Move Forgery Detection Using Block Representing Method", International Journal Of Soft Computing And Engineering (Ijsce) Issn: 2231-2307, Volume-4, Issue-2, May 2014

[10]. Sundaram. M And C. Nandini, "Feature Based Image Authentication Using Symmetric Surround Saliency Mapping In Image Forensics" International Journal Of Computer Applications (0975 - 8887) Volume 104 - No.13, October 2014

[11]. Y Yadav.P, Rathore.Y, Yadav.A,” Dwt Based Copymove Image Forgery Detection”, International Journal Of Advanced Research In Computer Science An Electronics Engineering Volume 1, Issue 5, July 2012

[12]. Ye.S, Sun.S And Chang.E, "Detecting Digital Image Forgeries By Measuring Inconsistencies Of Block Artifact”, Ieee, 2007 